\title{
CFD simulation of a heavy oil reservoir with AICV completion
}

\author{
M. Amaratunga ${ }^{1}$, K. K. Perera ${ }^{1}$, V. Mathiesen ${ }^{2} \&$ B. M. Halvorsen ${ }^{1}$ \\ ${ }^{I}$ Department of Process, Energy and Environmental Technology, \\ Telemark University College, Norway \\ ${ }^{2}$ Inflow Control AS, Norway
}

\begin{abstract}
Computational Fluid Dynamics (CFD) was used to analyze the flow behaviour of oil and water in the porous heavy oil reservoir with an Autonomous Inflow Control Valve (AICV) installed. 2D simulations in ANSYS FLUENT 13.0 for a $50 \mathrm{~m}$ long heavy oil reservoir section along the well were carried out with four AICVs placed on the main production pipe.

Two different cases were modelled by changing the volume fraction discritization scheme and the way of creating the pressure drop across the AICV. Both cases showed a successful imitation of AICV behaviour by automatically closing the valves completely for water and keeping them open for oil. The simulations performed under an implicit volume fraction scheme resulted in later breakthrough times and a diffused oil layer-like approach within the reservoir. The simulations performed under an explicit volume fraction scheme resulted in earlier breakthrough times and distinct flow behaviour of oil, which was identified as fingering phenomena. The oil production rates, breakthrough times, water migration within the reservoir and annulus were observed for both approaches. The accumulated oil production varies from 70 to $111.6 \mathrm{~m}^{3}$ due to different breakthrough times.
\end{abstract}

Keywords: CFD, heavy oil, implicit, explicit, discritization, breakthrough, $A I C V$.

\section{Introduction}

Economical production of heavy oil represents a major challenge for the current oil industry due to the presence of water, gas and sand in the reservoirs [1]. 
Fingering is an inherent problem to heavy oil and water systems. The disadvantage of the fingering is that, a lot of non produced oil can be bypassed due to water fingers and this may lead to reduced production and recovery from the oil reservoirs. The fingering phenomenon occurs due to the displacement of high viscous fluid by a low viscous fluid, where the water moves upward in higher velocities [2].

New technologies are important to increase the oil production and recovery. Inflow Control Devices (ICDs) are used to delay water and gas breakthrough and thereby increase the oil production and recovery [3]. The new technology, Autonomous Inflow Control Valve (AICV) is one of the latest developed technologies to prevent the water or gas getting into the production well [4].

Computational Fluid Dynamics (CFD) can be used to simulate the flow behaviour of oil, gas and water in oil reservoirs. The fundamental basis of CFD is the conservation laws of physics. The conservation laws are discritized using different calculation schemes over small spatial volumes in a fluid domain. For oil and water multiphase flow, the Eulerian multiphase model in ANSYS FLUENT is an appropriate model. This model solves one momentum equation and mass conservation equation for each phase [5].

This paper presents how the commercially available CFD software can be used to predict oil and water flow within the heavy oil reservoirs. The CFD model should be able to predict the viscous fingering effect. In addition the action of AICVs in heavy oil reservoirs is included in the model. The aim of the AICV in an oil reservoir with water drive is to close for water at breakthrough.

\section{Methodology}

2D simulations were performed to observe the behaviour of oil and water flow in a heavy oil reservoir with an underlying aquifer. Figure 1 presents the geometry of the reservoir and the location of the base pipe, the annulus, the packers and the valves. The base pipe was located on top of the oil reservoir and four identical rectangular shaped valves are installed at the bottom of the base pipe.

\subsection{Mesh generation}

The geometry shown in Figure 1 was plotted in GAMBIT 2.4 and meshed using "quad map" type with a high quality. The geometry consisted of four main regions which are reservoir, annulus, valves and base pipe. The reservoir, annulus and base pipe were defined as different zones and the four valves were defined as four individual zones. The valves are isolated from each other by using packers in the annulus region. The valve dimensions $0.08 \times 0.02 \mathrm{~m}^{2}$ were used in the simulations.

The pipe outlet was defined as a "pressure outlet" and the right end of the pipe was set as wall. The inlets of the four valves were set to interior to get the advantage of data acquisition. Three packers were introduced between the valves and they were set as wall boundaries to avoid the water flow emerging to the other valves through the annulus. The reservoir sides were defined as symmetry. 


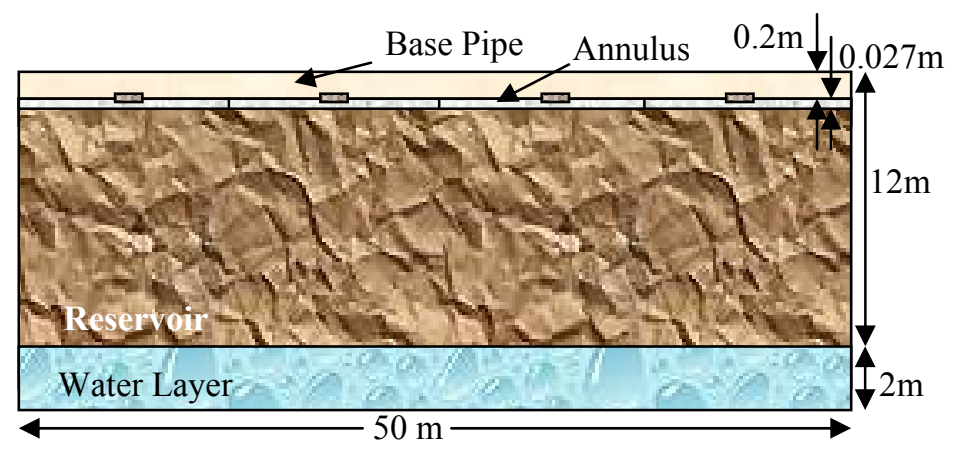

Figure 1: The defined geometry with dimensions.

\subsection{FLUENT set-up}

The imported geometry from GAMBIT was simulated under transient conditions. The multiphase Eulerian model was chosen and the viscous model was set to laminar. The pressure difference over the reservoir was taken as 5bars, while the reservoir porosity and permeability were set to 0.3 and $5 \mathrm{Da}$ respectively. The heavy oil viscosity was $100 \mathrm{cP}$ and the density was $970 \mathrm{~kg} / \mathrm{m}^{3}$. The water viscosity and the density were set to $1 \mathrm{cP}$ and $998 \mathrm{~kg} / \mathrm{m}^{3}$ respectively. The hydrostatic pressure was taken into account for determination of the pressure inlet from the bottom. Two cases with the same geometry were simulated in FLUENT.

A pressure drop along the base pipe creates a higher flow at the heel than in the toe. This is called the heel-toe effect. The pressure drop occurs due to the friction losses when the oil moves from the toe towards the heel. In a $3-4 \mathrm{~km}$ long horizontal well, the pressure difference along the pipe length can be significant. In the simulations, since the considered pipe length is only $50 \mathrm{~m}$, the pressure drop along the base pipe is very low. Therefore, approximations were used to restrict the flow and obtain a pressure drop through the base pipe. This is done to be able to study the water flow in the reservoir when the valves close at different time.

The characteristic of the AICV is that it closes for water and accepts only oil through it. The principle of the AICV is not included in the CFD model. Instead, a function is included that gives high restriction for water and very low restriction for oil through the valves. The necessary settings and the appropriate cell zone conditions for each case were adjusted to establish the flow restrictions through the valves in FLUENT. Two cases, Case A and Case B, were simulated using the volume fraction discritization scheme as implicit (Case A) and explicit (Case B), and the results were compared.

Phase coupled SIMPLE was used in both cases for the pressure velocity coupling and Modified HRIC was used in Case A for the volume fraction discritization while Geo-Reconstruct method was used in Case B. 


\section{Results and discussion}

The simulations were initialized in such way that the defined $2 \mathrm{~m}$ water layer was located at the bottom of the reservoir.

\subsection{Static pressure variation}

The objective of this study is to simulate the water breakthrough time and the distribution of oil and water in the reservoir after the AICV has locally closed for water. The pressure drop across each valve is approximately 1 bar. The restriction through the valves has been established in the same way in both cases. Figure 2 illustrates the pressure drops across each valve for the two cases. Figure 3 shows the static pressure distribution in the reservoir before and after the water breakthrough at Valve 1 in Case B. After water breakthrough the valve closes, and the pressure increases near the base pipe.

\subsection{Phase distributions within the reservoir}

Figure 4 shows the phase distribution within the reservoir in Case A and the movement of water through the annulus after the water breakthrough of all four valves. It can be seen that the water flows through the annulus until it reaches the packers. Then the packers restrict the flow beyond that point.

The heel-toe effect could be observed in both Case A and B. Figure 5 shows the phase distribution of oil and water in the reservoir after 4.7 days of simulation in Case B where the first water breakthrough occurs at valve 1.

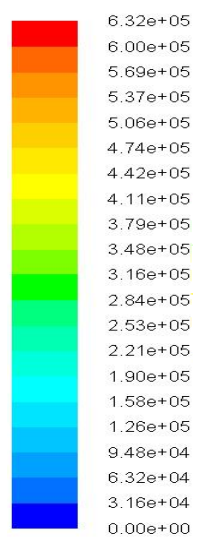

Figure 2:

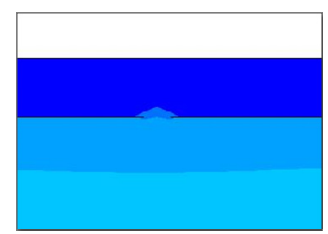

(a) Valve 1

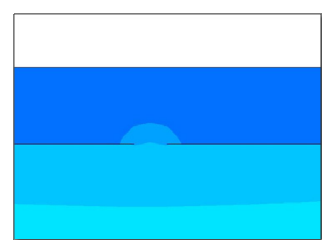

(c) Valve 3

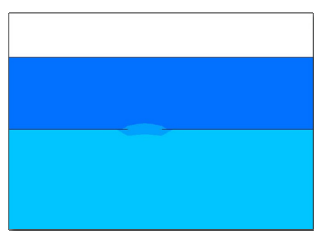

(b) Valve 2

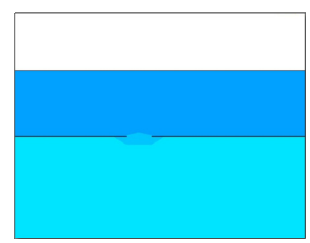

(d) Valve 4

Closer view of the pressure drops across each valve. 


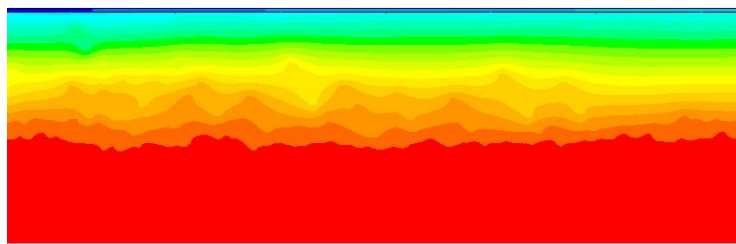

Before breakthrough from Valve 1

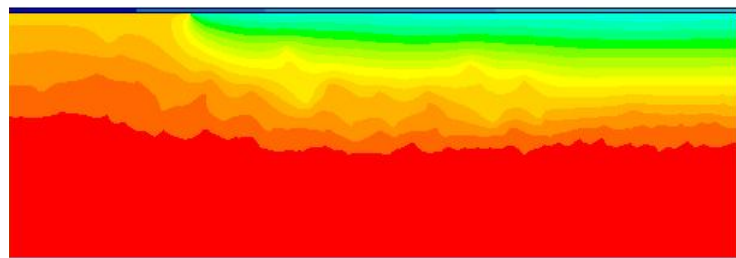

After breakthrough from Valve 1

Figure 3: $\quad$ Static pressure distribution of the system before and after the water breakthrough at valve 1 in Case B.
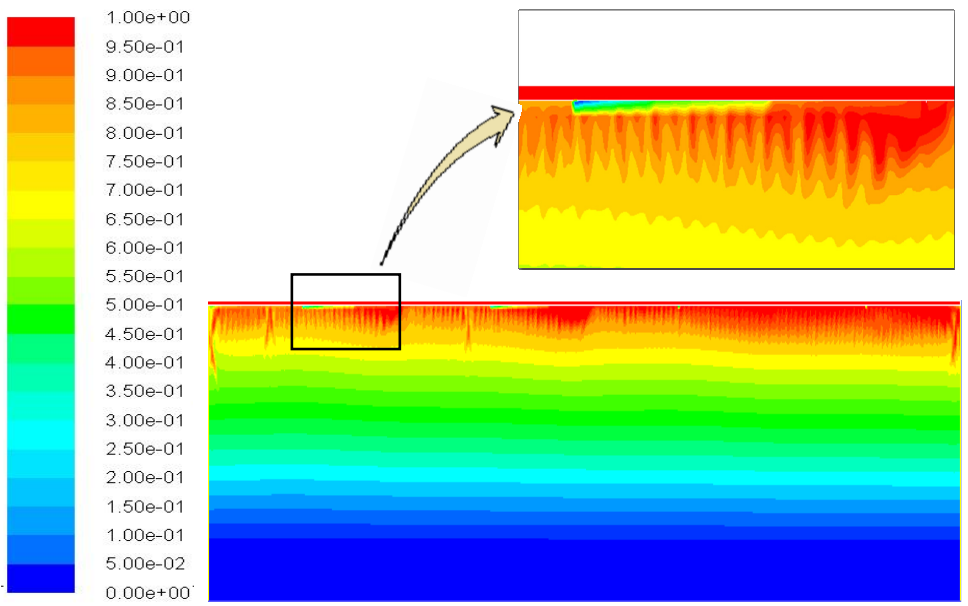

Figure 4: Phase distribution within the reservoir and water movement in the annulus, Case A. 


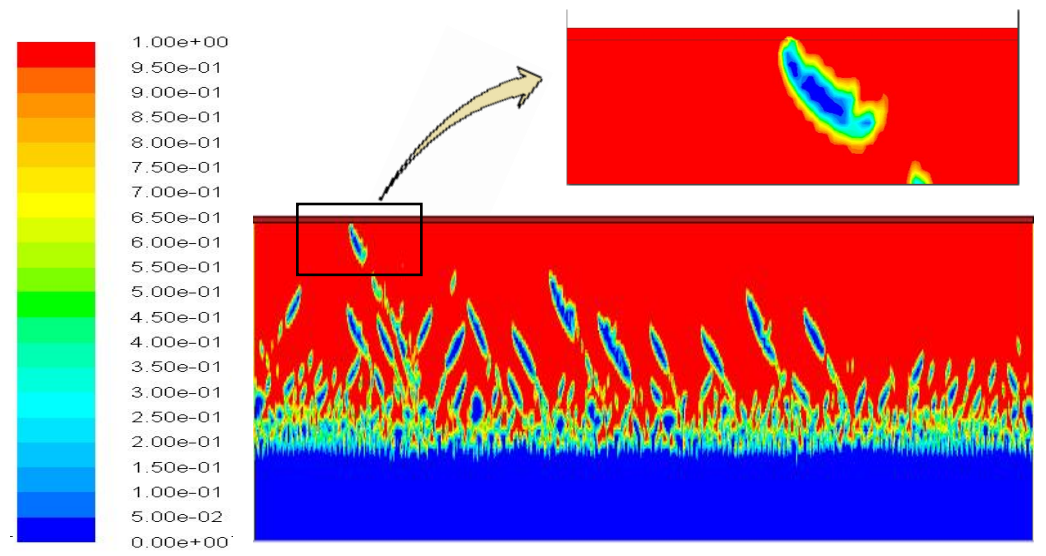

Figure 5: $\quad$ Phase distribution at water breakthrough in Valve 1 in Case B.

\subsection{Fingering phenomena and annular movement of water}

Viscous fingering is expected to occur in heavy oil reservoirs with water drive. It is therefore important to develop a CFD model that is able to predict the effect of fingering during oil production. In Case A, the water moves like a uniform layer towards the well. Case A predicts the heel-toe effect, the water breakthrough and the movement of water in the annulus and reservoir after the valves close. Case $\mathrm{B}$, which used the volume fraction discritization scheme, 'Geo-Reconstruct' under explicit scheme, is able to predict the intermediate transient flow behaviour within the reservoir which is identified as fingers. Figure 6 shows a situation where the water breakthrough has occurred at all valves in Case B and the movement of water within the annulus towards the packer.

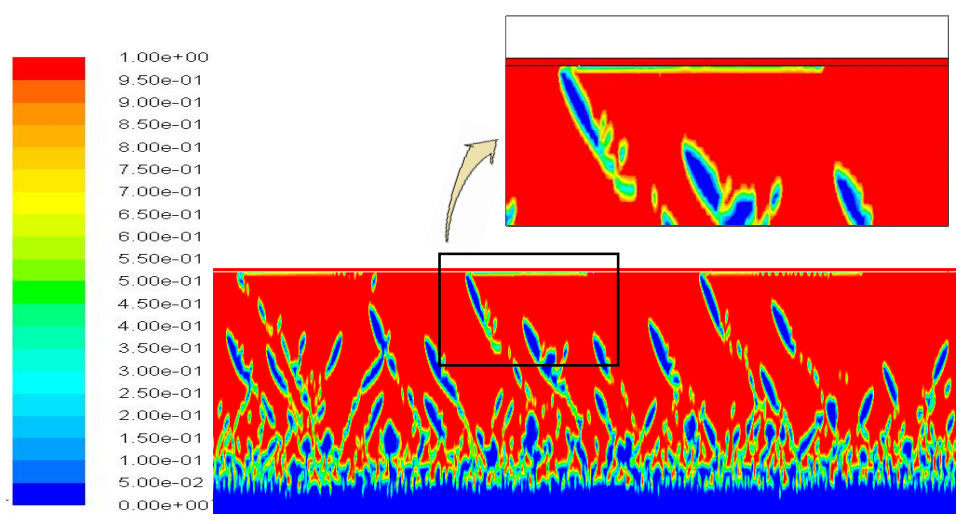

Figure 6: Phase distribution after 5.7 days and the annular movement of water after breakthrough. 
Packers were introduced into the annulus in order isolate the valves from one another, so that the water breakthrough at one valve cannot directly affect the others. The simulations could clearly predict the water behaviour through the annular region after the breakthrough. After the breakthrough, the valve is closed and there will not be any pressure drop over the valve. Hence the water loses its driving force and tends to flow through the annulus towards the next valve. The results indicate that the packers are able to stop the unwanted water flow to move into the neighbouring valve. The other zones of the well can then produce oil for a longer period.

\subsection{Breakthrough times and oil production}

The breakthrough times from each valve are shown in Figure 7. The breakthrough times in Case A are considerably higher than in Case B. The earlier breakthrough in Case B is due to the fingering effect which implies water fingers are moving towards the valves with a higher velocity than the oil. In Case A the water moves as a layer, and will reach the valves later. According to a study done by Wijeratne [6], it is found that the mesh size is very important to be able to simulate the fingering effect and the breakthrough time. The simulations in this study are performed with a very fine mesh, and should be able to predict the breakthrough times with good accuracy.

Figure 8 shows the oil production for Case A. The oil flow rate increases gradually and reaches the maximum when the breakthrough occurs. Then the valve closes and the oil and water production stops. The average oil production through a valve is approximately $4 \mathrm{~m}^{3} /$ day.

The production history of each valve for the case B is plotted in Figure 9. The average oil production through each valve is approximately $3.5 \mathrm{~m}^{3} /$ day which is slightly lower than in Case A. The fluctuating behaviour of the production through valve 4 can be due to the intermittent moisture blockage of valve 4 .

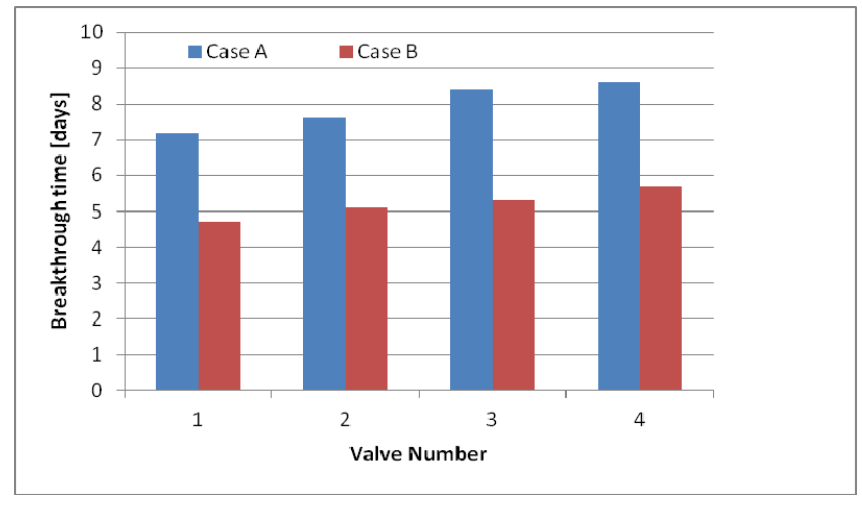

Figure 7: $\quad$ Breakthrough times for each valve. 


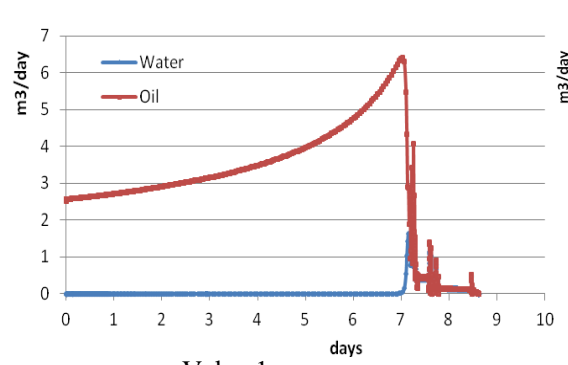

Valve 1

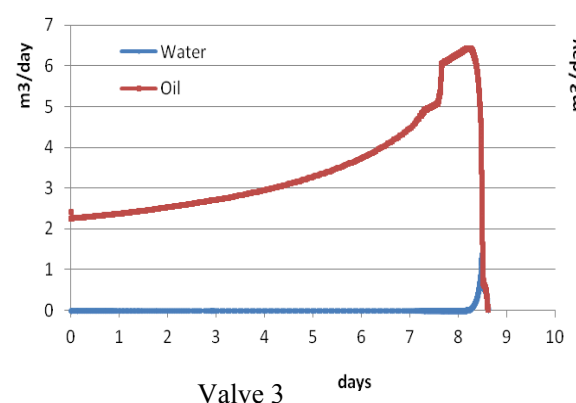

Valve 3 days

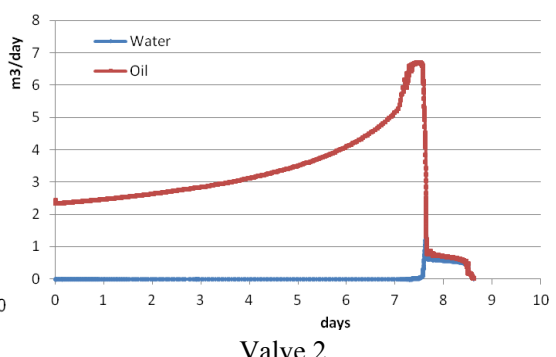

Valve 2

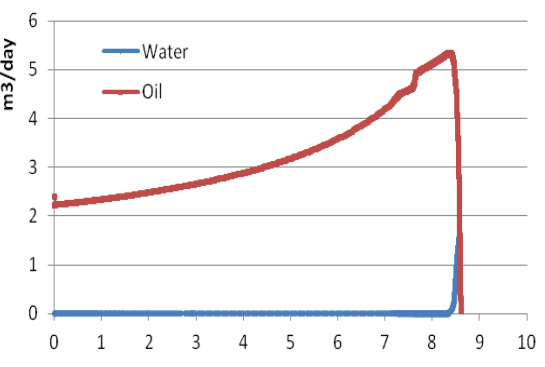

Valve 4 days

Figure 8: $\quad$ Oil production from each valve in Case A.

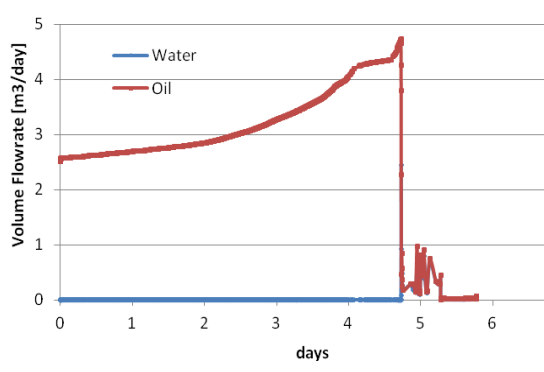

Valve 1

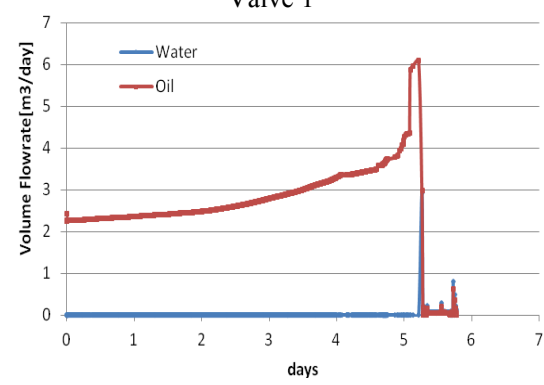

Valve 3

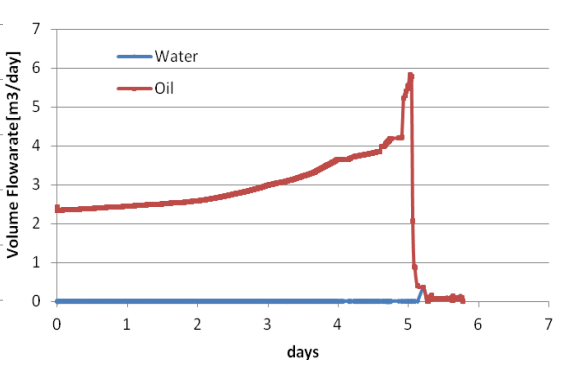

Valve 2

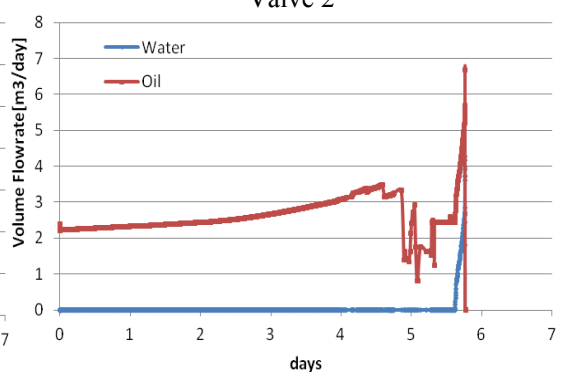

Valve 4

Figure 9: $\quad$ Oil production from each valve in Case B. 
According to the Figure 8 and Figure 9, the flow rates through the valves increase gradually until the breakthrough occurs. This implies that the valve pressure drop also has to increase with time. This is realistic since the oil column is decreasing with time and hence the pressure drop due to friction in the reservoir is reduced equivalently.

The most important feature of the AICV is that it autonomously shut-off the water at breakthrough. This was achieved in both cases of the simulations. There is a sudden rise of oil flow rate when the breakthrough occurs and then it tends to fall down with the rise of water production as shown in Figure 8 and Figure 9 The oil production is continued to drop down until the valve has automatically closed for water. Hence this study has successfully simulated the valve action to get the valve closed at the water breakthrough. Figure 10 shows accumulated oil production for the two cases of simulation. As it was mentioned earlier, the explicit case has resulted in lower breakthrough times and because of that the accumulated oil production is lower compared to Case A. The curves represent the oil production until all the four valves are closed.

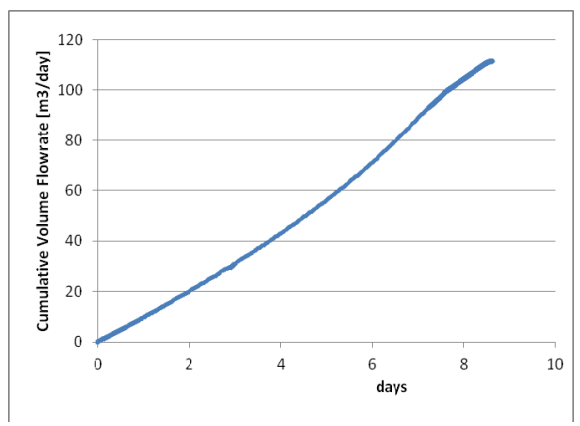

Case A

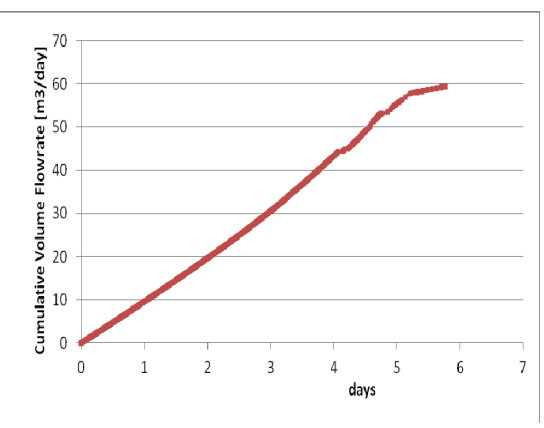

Case B

Figure 10: $\quad$ Accumulated oil production for the two cases.

\section{Conclusion}

Horizontal well technology has been identified as an efficient way of producing heavy crude oil. The use of inflow control devices can increase the oil production and recovery. AICV is a patented new technology that can shut-off the water locally at water breakthrough.

The AICV valve action was successfully simulated in $2 \mathrm{D}$ simulations in FLUENT where the valves were automatically closed for water. The inherent fingering phenomena could be seen in case B simulated under the explicit volume fraction discritization scheme. Case A with the implicit scheme did not predict the fingering phenomenon.

The breakthrough times were different for the two cases and it was influenced by the volume fraction scheme parameter (implicit or explicit). The packers are 
good obstacles to isolate the valves and avoid the water flowing from one valve to the other after the water breakthrough. It is recommended to use the Geo Reconstruct discritization under explicit scheme to observe the distinct intermediate phase separations in the future studies.

\section{References}

[1] S. Saskoil and R. Butler, "The production of conventional heavy oil reservoirs with bottom water using steam-assisted gravity drainage," Journal of Canadian Petroleum Technology, vol. 29, 1990.

[2] G. M. Homsy, "Viscous Fingering in Porous Media," Annual Review of Fluid Mechanics, vol. 19, pp. 271-311, 1987.

[3] L.-B. Ouyang, "Practical consideration of an inflow-control device application for reducing water production," in SPE Annual Technical Conference and Exhibition, 2009.

[4] H. Aakre, B. Halvorsen, B. Werswick and V. Mathiesen, "Smart Well With Autonomous Inflow Control Valve Technology," in 18th Middle East Oil \& Gas Show and Conference (MEOS), 2013.

[5] I. Ansys. (2010). ANSYS FLUENT 13.0 user's guide.

[6] Wijeratne D.I.E.N., "Inflow Control and packers in horizontal oil wells," Master Thesis, Department of process, energy and environment Technology, Telemark University College, Norway, 2011. 